\title{
Global stability of the error dynamics of an observer designed for the slow states of a singularly perturbed system
}

\author{
Luis Cuevas, Dragan Nešić and Chris Manzie
}

\begin{abstract}
In this note, we study the stability of the error dynamics of an observer designed to estimate only the slow states of a singularly perturbed system. The observer is designed on the basis of the reduced (slow) model. We have recently reported semi-global practical results for this problem. Our previous work can be used to state local and regional convergence of the estimation error, but we cannot conclude global results from it. We seek to prove a stronger (global) result under stronger (global) assumptions in this manuscript. Moreover, we focus on proving the robustness of an observer with respect to singular perturbations and with respect to the measurement noise.
\end{abstract}

\section{INTRODUCTION}

Observer design for singularly perturbed nonlinear systems is a hard task to address. Estimation of the slow states of a two-time scale system via an observer designed for the slow system is a common approach in the singular perturbation framework, see [1]-[4]. It was shown in [1] that under a set of appropriate assumptions the estimation error is semi-globally practically asymptotically stable for a general class of plants and observers. These results were illustrated through practical applications in [2].

Estimation of slow states via a nonlinear Luenberger-type observer is presented in [3]. The observer designed for the reduced system exhibits a linear error dynamics. It is shown a local convergence of the estimation error when the observer is implemented on the full system. A Luenberger-type observer to estimate the slow and fast states of a particular class of plants is reported in [5]. The observer gains are presented in terms of an LMI condition which is independent of the perturbation parameter and that guarantees exponential stability of the error dynamics. Note that these results cannot be extended to cover a larger class of plants. Another work on the topic is presented in [4] in which a sliding mode observer for the slow states is designed. Note that results in [3]-[5] deal with specific plants and specific observers.

Here, we estimate the slow states of a singularly perturbed plant by using an observer designed for the reduced system and implemented on the original plant. We consider a general class of plants with measurement noise and study the robustness of the observer with respect to it. To the best of our knowledge, no current results address this robustness analysis in systems with two-time scales. We assume input-to-state

This work was supported by the Melbourne International Research Scholarship scheme of The University of Melbourne, and by the Australian Research Council Discovery Project.

L. Cuevas, D. Nešić, and C. Manzie are with the Department of Electrical and Electronic Engineering and belong to the MIDAS Laboratory, The University of Melbourne, Parkville, VIC 3010, Australia. lcuevas@student.unimelb.edu.au, dnesic@unimelb.edu.au, manziec@unimelb.edu.au practical stability (ISpS) for the reduced system and global exponential stability for the boundary layer system. By assuming that the error dynamics for the reduced system are input-to-state stable (ISS), we prove the error dynamics are ISpS stable when the observer is used on the original plant. Moreover, we exploit our assumptions to state robustness results on finite-gain $\mathcal{L}_{2}$ stability. We present two classes of systems and observers for which our results hold. The considered plants have reduced systems with the appropriate structure to design a class of high-gain observers [6] and a class of circle criterion-based $\mathcal{H}_{\infty}$ observers [7].

In addition to our main contribution, our assumptions lead to stronger conclusions in the absence of measurement noise than those in [1]. When there are no disturbances to the system, our main result implies practical global exponential stability of the error dynamics. Although we can apply results in [1] to the problem presented in this paper, the proofs in there would lead to weaker conclusions. Here, we state a global stability property for the error dynamics, while [1] only allows to conclude semi-global results, and local and regional results under slightly different assumptions.

Notation: The (Euclidean) norm of a vector $x \in \mathbb{R}^{n}$ is denoted as $|x|$. We say that $s \in \mathcal{L}_{\infty}$ if $|s|_{\infty}<\infty$, where $|s|_{\infty}:=\operatorname{ess}_{\sup }|s(t)|$. The minimum eigenvalue of a square matrix $A$ is denoted by $\lambda_{\min }\{A\}$. We use the notation $\left|s\left[t_{1}, t_{2}\right]\right|:=\sup _{t \in\left[t_{1}, t_{2}\right]}|s(t)|$. The $\mathcal{L}_{2}$-norm of a signal is defined as $|s(t)|_{\mathcal{L}_{2}}=\sqrt{\int_{0}^{\infty} s(t)^{T} s(t) d t}$.

\section{Problem Statement}

Consider a class of plants in the singular perturbation standard form given by

$$
\begin{aligned}
\dot{x} & =f_{s}(t, x, z, u, \epsilon), \\
\epsilon \dot{z} & =f_{f}(t, x, z, u, \epsilon), \\
y & =h(t, x, z, u, w, \epsilon),
\end{aligned}
$$

where $x \in \mathbb{R}^{n}$ represents the slow state variables, $z \in \mathbb{R}^{m}$ is the fast state of the system, $y \in \mathbb{R}^{p}$ is the measured output, $u \in \mathbb{R}^{r}$ is the input, $w \in \mathbb{R}^{q}$ is measurement noise and $\epsilon>0$ is the perturbation parameter. Note that a major difference with [1] is the inclusion of the measurement noise in (1c). Assumption 1. The input of the system and its derivative belong to $\mathcal{L}_{\infty}$; i.e. $u, \dot{u} \in \mathcal{L}_{\infty}$.

Our goal is to analyse the performance of an observer designed for the reduced system of a singularly perturbed system and implemented on the original plant. Hence, the robustness of the observer with respect to singular perturbations is tested. Following the standard singular perturbation 
technique, we set $\epsilon=0$ to obtain the algebraic equation

$$
0=f_{f}(t, x, z, u, 0) \text {. }
$$

Assumption 2. The algebraic equation (2) has an isolated solution $z=H(t, x, u)$ that can be obtained analytically. Now, substitute the isolated solution $z=H(t, x, u)$ in (1a) and (1c) at $\epsilon=0$ to obtain the reduced dynamical system

$$
\begin{aligned}
\dot{x} & =f_{s}(t, x, H(t, x, u), u, 0), \\
y_{s} & =h(t, x, H(t, x, u), u, 0) .
\end{aligned}
$$

Assumption 3. For the reduced (slow) system (3), there exists a continuously differentiable function $V_{1}(t, x)$, positive constants $b_{i}(i=1, \ldots, 5)$, and $\delta_{V_{1}} \geq 0$ such that for all $x \in \mathbb{R}^{n}, u \in \mathbb{R}^{n}, t \geq 0^{1}$

$$
\begin{aligned}
b_{1}|x|^{2} & \leq V_{1}(t, x) \leq b_{2}|x|^{2}, \\
\frac{\partial V_{1}}{\partial t}+\frac{\partial V_{1}}{\partial x} f_{s}(t, x, H, u, 0) & \leq-b_{3}|x|^{2}+b_{4}|u|^{2}+\delta_{V_{1}}, \\
\left|\frac{\partial V_{1}}{\partial x}\right| & \leq b_{5}|x| .
\end{aligned}
$$

Our assumptions in here are stronger than their counterpart in [1]. Here, we exploit these strong conditions to conclude stronger results than those in [1]. Consider the change of variables $\xi=z-H(t, x, u)$ and note that (1) becomes

$$
\begin{aligned}
\dot{x} & =f_{s}(t, x, \xi+H(t, x, u), u, \epsilon), \\
\epsilon \dot{\xi} & =f_{f}(t, x, \xi+H(t, x, u), u, \epsilon)-\epsilon \frac{\partial H}{\partial t} \\
& -\epsilon \frac{\partial H}{\partial x} f_{s}(t, x, \xi+H(t, x, u), u, \epsilon)-\epsilon \frac{\partial H}{\partial u} \dot{u}, \\
y & =h(t, x, \xi+H(t, x, u), u, \epsilon) .
\end{aligned}
$$

We analyse the fast dynamics behaviour by studying the system (7b) in the fast time scale $\tau=\frac{t-t_{0}}{\epsilon}$ as follows

$$
\begin{aligned}
\frac{d \xi}{d \tau} & =f_{f}(t, x, \xi+H(t, x, u), u, \epsilon)-\epsilon \frac{\partial H}{\partial t} \\
& -\epsilon \frac{\partial H}{\partial x} f_{s}(t, x, \xi+H(t, x, u), u, \epsilon)-\epsilon \frac{\partial H}{\partial u} \dot{u},
\end{aligned}
$$

where we have used the approach in [8]. Set $\epsilon=0$ and treat $t$ and $x$ as constants to obtain the boundary layer system

$$
\frac{d \xi}{d \tau}=f_{f}(t, x, \xi+H(t, x, u), u, 0) .
$$

Assumption 4. There exists a Lyapunov function $W(t, x, \xi)$ and positive constants $c_{i},(i=1, \ldots, 6)$ such that for all $(x, \xi) \in \mathbb{R}^{n} \times \mathbb{R}^{m}, u \in \mathbb{R}^{r}$ and $t \geq 0$

$$
\begin{gathered}
c_{1}|\xi|^{2} \leq W(t, x, \xi) \leq c_{2}|\xi|^{2} \\
\frac{\partial W}{\partial \xi} f_{f}(t, x, \xi+H(t, x, u), u, 0) \leq-c_{3}|\xi|^{2} \\
\left|\frac{\partial W}{\partial \xi}\right| \leq c_{4}|\xi|, \quad\left|\frac{\partial W}{\partial t}\right| \leq c_{5}|\xi|^{2}, \quad\left|\frac{\partial W}{\partial x}\right| \leq c_{6}|\xi| .
\end{gathered}
$$

Remark 1. Even though Assumption 4 is strong due to the uniformity in $u$ in (11), it has been used before in

\footnotetext{
${ }^{1}$ In the sequel, when it is necessary, we would suppress arguments of some functions to simplify the notation.
}

singular perturbations analysis [8]. If we claim that (9) is exponentially stable uniformly in $t, x$ and $u$, we can use the converse Lyapunov theorem [Lemma 9.8, 9] in which is stated $\left|\frac{\partial W}{\partial x}\right| \leq c|\xi|^{2}$ for some $c>0$. This is a slightly more general condition than the last inequality in (12). However, we need condition (12) for our proof to state a global result.

\section{Practical DiSS and Practical $\mathcal{L}_{2}$ Stability of THE ORIGINAL PLANT}

We first study the boundedness of (7) to establish our main result. To perform this analysis, we consider terms that arise due to the interconnection of the slow and fast dynamics. These terms are sign indefinite, so we need to bound them. Assumption 5. Consider $f_{s}(t, x, \xi+H, u, \epsilon)$ and let $L_{i}$ $(i=0,1,2)$ be nonnegative constants such that the following inequalities hold for all $x \in \mathbb{R}^{n}, \xi \in \mathbb{R}^{m}, u \in \mathbb{R}^{r}$, and $t \geq 0$

$$
\begin{gathered}
\left|f_{s}(t, x, \xi+H, u, \epsilon)-f_{s}(t, x, \xi+H, u, 0)\right| \leq \epsilon L_{0}(|x| \\
+|\xi|+|u|) \\
\left|f_{s}(t, x, \xi+H, u, 0)-f_{s}(t, x, H, u, 0)\right| \leq L_{1}|\xi| \\
\left|f_{s}(t, x, H, u, 0)\right| \leq L_{2}(|x|+|u|) .
\end{gathered}
$$

Assumption 6. Consider $f_{f}(t, x, \xi+H, u, \epsilon)$ and $H(t, x, u)$. Let $L_{i}(i=3, \ldots, 6)$ be nonnegative constants such that the following set of inequalities holds for all $x \in \mathbb{R}^{n}, \xi \in \mathbb{R}^{m}$, $u \in \mathbb{R}^{r}$, and $t \geq 0$

$$
\begin{aligned}
& \left|f_{f}(t, x, \xi+H, u, \epsilon)-f_{f}(t, x, \xi+H, u, 0)\right| \leq \epsilon L_{3}(|x| \\
& +|\xi|+|u|) \text {, } \\
& \left|\frac{\partial H}{\partial t}\right| \leq L_{4}(|x|+|u|), \quad\left|\frac{\partial H}{\partial x}\right| \leq L_{5}, \quad\left|\frac{\partial H}{\partial u}\right| \leq L_{6} .
\end{aligned}
$$

Remark 2. Inequalities in Assumptions 5 and 6 can be deduced from general conditions over $f_{s}, f_{f}$ and $H$. If $f_{s}$ and $f_{f}$ are continuously differentiable with bounded derivatives, $f_{s}(0,0,0,0, \epsilon)=0, f_{f}(0,0,0,0, \epsilon)=0$ and $H(t, 0,0)=0$, we can conclude (13) - (16). Conditions in (17) can be deduced if $\partial H / \partial x$ and $\partial H / \partial u$ have continuous and bounded first partial derivatives with respect to $t$ [11].

Lemma 1. Consider the singularly perturbed system (7). If Assumptions 1-6 hold, there exists $\tilde{\epsilon}^{*}>0, k_{i}>0(i=1,2)$, $k_{i} \geq 0(i=3, \ldots, 7), \ell_{1}>0, \ell_{i} \geq 0(i=2, \ldots, 6)$ such that the system (7) satisfies the following ISS and $\mathcal{L}_{2}$ conditions $^{2}$

$$
\begin{aligned}
|(x(t), \xi(t))| \leq & k_{1} \exp \left[-k_{2}\left(t-t_{0}\right)\right]\left|\left(x_{0}, \xi_{0}\right)\right|+\left(k_{3}+\epsilon^{\frac{1}{2}} k_{4}\right. \\
+ & \left.\epsilon k_{5}\right)\left|u\left[t_{0}, t\right]\right|+\epsilon^{\frac{1}{2}} k_{6}\left|\dot{u}\left[t_{0}, t\right]\right|+k_{7}, \\
|(x(t), \xi(t))|_{\mathcal{L}_{2}} & \leq \ell_{1}\left|\left(x_{0}, \xi_{0}\right)\right|+\left(\ell_{2}+\epsilon^{\frac{1}{2}} \ell_{3}+\epsilon \ell_{4}\right)|u(t)|_{\mathcal{L}_{2}} \\
& +\epsilon^{\frac{1}{2}} \ell_{5}|\dot{u}(t)|_{\mathcal{L}_{2}}+\ell_{6} t,
\end{aligned}
$$

for all $\epsilon \in\left(0, \tilde{\epsilon}^{*}\right)$ and for all $\left(x_{0}, \xi_{0}\right) \in \mathbb{R}^{n} \times \mathbb{R}^{m}$, $u, \dot{u} \in \mathcal{L}_{\infty}$, and $t \geq 0$. Furthermore, there exists $\bar{\epsilon}^{*}>0$, $\tilde{k}_{i} \geq 0(i=1,2), \tilde{k}_{i} \geq 0(i=3,4,5), \tilde{\ell}_{1}>0$ and $\tilde{\ell}_{i} \geq 0$

\footnotetext{
${ }^{2}$ In the sequel, $x_{0}:=x(0)$. The same apply for the other states.
} 
$(i=2, \ldots, 5)$ such that the fast states satisfy the following ISS and $\mathcal{L}_{2}$ conditions

$$
\begin{aligned}
|\xi(t)| \leq & \tilde{k}_{1} \exp \left(-\tilde{k}_{2} \frac{t-t_{0}}{\epsilon}\right)\left|\xi_{0}\right|+\left(\epsilon \tilde{k}_{3}+\epsilon^{2} \tilde{k}_{4}\right)\left(\left|x\left[t_{0}, t\right]\right|\right. \\
& \left.+\left|u\left[t_{0}, t\right]\right|\right)+\epsilon \tilde{k}_{5}\left|\dot{u}\left[t_{0}, t\right]\right| \\
|\xi(t)|_{\mathcal{L}_{2}} & \leq \epsilon^{\frac{1}{2}} \tilde{\ell}_{1}\left|\xi_{0}\right|+\epsilon\left(\tilde{\ell}_{2}+\epsilon \tilde{\ell}_{4}\right)|x(t)|_{\mathcal{L}_{2}}+\epsilon \tilde{\ell}_{3}|\dot{u}(t)|_{\mathcal{L}_{2}} \\
& +\epsilon\left(\tilde{\ell}_{2}+\epsilon \tilde{\ell}_{5}\right)|u(t)|_{\mathcal{L}_{2}},
\end{aligned}
$$

for all $\epsilon \in\left(0, \bar{\epsilon}^{*}\right), \xi_{0} \in \mathbb{R}^{m}, x, u, \dot{u} \in \mathcal{L}_{\infty}$, and $t \geq 0$.

Remark 3. We have taken advantage of our assumptions to prove ISS and $\mathcal{L}_{2}$ stability results for the singularly perturbed plant. Lemma 1 states a practical DISS result for the full state and the fast variable. It is observed that if we set $\epsilon=0$ in (18) we recover the property implied by Assumption 3. The $\mathcal{L}_{2}$ results are equivalent to the ISS ones but from the perspective of finite-gain $\mathcal{L}$ stability. Note that $\mathcal{L}_{2}$ bounds are very useful when dealing with $\mathcal{L}_{2}$ bounded noise and in optimisation problems. Both ISS and $\mathcal{L}_{2}$ conditions prove different robustness properties of the system which are used to conclude appropriate results in our main theorem.

\section{MAIn RESUlT}

We now analyse the robustness of a full-order nonlinear observer designed for the reduced system (3) and implemented on the full system (7). We give a general set of assumptions that cover a large class of observers to state global stability results in the presence of measurement noise. Our results imply that the error dynamics is globally practically stable in the absence of measurement noise in which the practical term can be made small by reducing $\epsilon$.

Consider the class of full-order observers described by

$$
\dot{\hat{x}}=f_{o}\left(t, \hat{x}, y_{s}, u\right)
$$

where $\hat{x} \in \mathbb{R}^{n}$ is the state of the observer and an estimate of $x \in \mathbb{R}^{n}$ (slow variable), $y_{s}$ and $u$ are the output and input of the nonlinear reduced system (3). Since the observer dynamics (22) has the same structure as the one we considered in [1], our stronger assumptions imply we work with a subclass of the observers covered by [1]. Define the estimation error as $e=x-\hat{x}$. Therefore, the error dynamics for the observer designed and implemented in the slow system (3) is given by

$$
\dot{e}=f_{e}\left(t, x, e, H(t, x, u), y_{s}, u, 0\right) .
$$

where $f_{e}=f_{s}(t, x, H, u, 0)-f_{o}\left(t, x-e, y_{s}, u\right)$.

Assumption 7. For the error dynamics in (23), there exists a continuously differentiable function $V_{e}(t, e)$, and positive constants $a_{1}, a_{2}, a_{3}, a_{4}$ and $a_{5}$, such that for all $x, e \in \mathbb{R}^{n}$, $u \in \mathbb{R}^{r}, w \in \mathbb{R}^{q}, t \geq 0$

$$
\begin{gathered}
a_{1}|e|^{2} \leq V_{e}(t, e) \leq a_{2}|e|^{2} \\
\frac{\partial V_{e}}{\partial t}+\frac{\partial V_{e}}{\partial e} f_{e}\left(t, x, e, H, y_{s}, u, 0\right) \leq-a_{3}|e|^{2}+a_{4}|w|^{2} \\
\left|\frac{\partial V_{e}}{\partial e}\right| \leq a_{5}|e|
\end{gathered}
$$

From condition (25), we can conclude ISS with respect to the measurement noise with an exponential $\mathcal{K} \mathcal{L}$ function and linear gain. Moreover, since we consider a quadratic term for $w$, an $\mathcal{L}_{2}$ stability property with linear gain is implied too.

The observer designed for the slow system (3) must be implemented on the original system (1). Due to the influence of the the perturbation parameter $\epsilon$ and the fast state $\xi$, the error dynamics is now given by

$$
\dot{e}=f_{e}(t, x, e, \xi+H, y, u, \epsilon),
$$

where $f_{e}=f_{s}(t, x, \xi+H, u, \epsilon)-f_{o}(t, x-e, y, u)$. Note that the extended state $(x, e, \xi)$ represents the interconnection between the system (7) and the error dynamics in (27). So, the full extended interconnected system is given by

$$
\begin{aligned}
\dot{x} & =f_{s}(t, x, \xi+H(t, x, u), u, \epsilon), \\
\dot{e} & =f_{e}(t, x, e, \xi+H(t, x, u), y, u, \epsilon), \\
\epsilon \dot{\xi} & =f_{f}(t, x, \xi+H(t, x, u), u, \epsilon)-\epsilon \frac{\partial H}{\partial t} \\
& -\epsilon \frac{\partial H}{\partial x} f_{s}(t, x, \xi+H(t, x, u), u, \epsilon)-\epsilon \frac{\partial H}{\partial u} \dot{u}, \\
y & =h(t, x, \xi+H(t, x, u), u, \epsilon) .
\end{aligned}
$$

To obtain the robustness result that we are looking for, we need to analyse (28). To prove our main results, we take advantage of the cascade properties of the system. We use a Lyapunov approach to generate an estimate of the upper bound of the estimation error; then, we use ISS and $\mathcal{L}_{2}$ approaches to conclude Theorem 1.

Assumption 8. Let $L_{7}$ and $L_{8}$ be nonegative constants such that $f_{o}(t, x-e, y, u)$ satisfies the following inequality for all $x \in \mathbb{R}^{n}, \xi \in \mathbb{R}^{m}, u \in \mathbb{R}^{r}, w \in \mathbb{R}^{q}$, and $t \geq 0$

$\left|f_{o}(t, x-e, y, u)-f_{o}\left(t, x-e, y_{s}, u\right)\right| \leq \epsilon L_{7}+L_{8}|\xi|$,

where $y=h(t, x, \xi+H, u, w, \epsilon)$ and $y_{s}=h(t, x, H, u, w, 0)$. Remark 4. Equation (29) can be obtained if $h(t, x, z, u, \epsilon)$ is globally Lipschitz in $(x, z, u, \epsilon)$ uniformly in $t$ and if $f_{o}(t, \hat{x}, y, u)$ is globally Lipschitz in $(\hat{x}, y, u)$ uniformly in $t$. Theorem 1. Consider the singularly perturbed system (28). If Assumptions $1-8$ hold, there exists $\epsilon^{*}>0, \bar{k}_{i}>0(i=1,2)$, $\bar{k}_{i} \geq 0(i=3, \ldots, 6), \hat{\ell}_{1}>0$ and $\hat{\ell}_{i} \geq 0(i=2, \ldots, 5)$ such that the error dynamics satisfy the following ISS and $\mathcal{L}_{2}$ stability properties

$$
\begin{aligned}
& |e(t)| \leq \bar{k}_{1} \exp \left[-\bar{k}_{2}\left(t-t_{0}\right)\right]\left|e_{0}\right|+\epsilon \bar{k}_{3}+\epsilon \bar{k}_{4}\left[\left|x\left[t_{0}, t\right]\right|\right. \\
& \left.+\left|\xi\left[t_{0}, t\right]\right|+\left|u\left[t_{0}, t\right]\right|\right]+\bar{k}_{5}\left|\xi\left[t_{0}, t\right]\right|+\bar{k}_{6}\left|w\left[t_{0}, t\right]\right| \\
& |e(t)|_{\mathcal{L}_{2}} \leq \hat{\ell}_{1}\left|e_{0}\right|+\hat{\ell}_{2}|w(t)|_{\mathcal{L}_{2}}+\epsilon \hat{\ell}_{3}\left[|x(t)|_{\mathcal{L}_{2}}+|\xi(t)|_{\mathcal{L}_{2}}\right. \\
& \left.+|u(t)|_{\mathcal{L}_{2}}\right]+\epsilon \hat{\ell}_{4} t+\hat{\ell}_{5}|\xi(t)|_{\mathcal{L}_{2}}
\end{aligned}
$$

for all $\epsilon \in\left(0, \epsilon^{*}\right), e_{0} \in \mathbb{R}^{n}, x, \xi, u, \dot{u} \in \mathcal{L}_{\infty}$ and $t \geq 0$.

Remark 5. The error dynamics are in cascade with the state $(x, \xi)$. Hence, $x(t)$ and $\xi(t)$ are seeing as signals in Theorem 1. It follows from Lemma 1 that $x(t)$ and $\xi(t)$ are bounded signals when considered as inputs to the error dynamics for any initial conditions and bounded inputs with 
bounded derivatives. By (20), the fast states rapidly converge to a ball of order $\epsilon$ since the exponential function in (20) quickly converges to zero in a finite time $T_{\xi}>0$ that depends on $\xi_{0}$. Note that the convergence rate can be adjusted by reducing $\epsilon$. Then, it can be proven that the $O(1)$ term in $\epsilon$ that depends on $\xi(t)$ in (30) rapidly converges. It is observed in (30) that the gains from $x$ to $e$ are of $O(\epsilon)$. Then, the effect of $x$ over the error dynamics can be attenuated by reducing $\epsilon$. Moreover, Lemma 1 implies there is a finite time $T>0$ depending on $\left(x_{0}, \xi_{0}\right)$ such that the contribution due to $x$ becomes of $O(\epsilon)$ for all $t \geq T$. Hence, for all $t>T$ the ultimate bound for the estimation error is an $O(\epsilon)$ term plus the input gain due to the measurement noise. Note that the $O(\epsilon)$ in the ultimate bound only depends on $\bar{k}_{3}, u$ and $\dot{u}$. By using an ISS approach for interconnected systems as in [Lemma 4.7, 9], it can be shown that (30) can be written in a form such that if $\epsilon$ is arbitrarily small, the property implied by Assumption 7 is recovered.

Remark 6. Theorem 1 implies "practical" $\mathcal{L}_{2}$ stability, where the practical meaning is understood based on the definition [Property $\left.I_{3}, 10\right]$. Note that the practical term in (31) is of $O(\epsilon)$. By using the cascade properties of the error dynamics, it can be proven that (31) has an equivalent form in which the input gains from $u, \dot{u}$ and $\left(x_{0}, \xi_{0}\right)$ to $e$ are of $O(\epsilon)$. Moreover, Lemma 1 implies the gain from $\xi_{0}$ to $e$ is of $O\left(\epsilon^{\frac{1}{2}}\right)$. It follows that we can find a finite time $T>0$ such that the $\mathcal{L}_{2}$ bound becomes a term of $O(\epsilon)$ plus the contribution due to the measurement noise. Since the contribution from the disturbance to the error dynamics has a finite gain in (31), it implies that the observer is robust with respect to measurement noise when the described approach is used.

Remark 7. In the absence of the measurement noise, Theorem 1 implies global exponential practical stability of the error dynamics which is a stronger conclusion than what [1] can provide.

\section{Applications}

Even though our results are not constructive, we have given conditions to cover a large class of plants and observers as illustrated here on two examples of high-gain observers and circle criterion-based $\mathcal{H}_{\infty}$ observers. We show that the stated assumptions are satisfied on these examples. This demonstrates that our results apply to existing nonlinear observer design methods in the literature.

\section{A. High-Gain Observer}

We restrict the class of plants such that the reduced system has the structure for which we can apply the observer in [6]. We choose a class of systems that is covered by the general class of plants (1). Consider plants with the following form

$$
\begin{aligned}
\dot{x} & =A x+f(t, x, z) \\
\epsilon \dot{z} & =M_{1} x+M_{2} z, \\
y & =C_{1} x+C_{2} z+D w,
\end{aligned}
$$

where $x \in \mathbb{R}^{n}$ and $z \in \mathbb{R}^{m}$ are the slow and fast state vectors, $y \in \mathbb{R}^{p}$ is the measured output, $w(t) \in \mathbb{R}^{p}$ is the measurement noise which is assumed to be globally bounded, $\epsilon$ is the perturbation parameter of the plant, and $A, C_{1}, C_{2}$, $D, M_{1}$ and $M_{2}$ are matrices of appropriate dimensions.

Assumption 9. The function $f(t, x, z)$ is continuous, globally Lipschitz and $f(t, 0,0)=0$.

Assumption 10. The matrix $M_{2}$ is Hurwitz.

We require a linear dynamics in (32b) for two reasons: 1) it is easier to compute the slow manifold, and 2) after the model reduction the slow system has the structure for which we can design the observer [6]. Assumption 10 is required to approximate the system via the lower dimensional systems.

Next, we demonstrate that Assumptions 1 - 5 are satisfied for the class of systems (32) so that we can use conclusions of Lemma 1 for this class of plants. Then, we introduce the observer and show that Assumptions 7 and 8 hold, which implies we can use results in Theorem 1. Note that there are no inputs to the system, then Assumption 1 trivially holds.

We now set $\epsilon=0$ in (32b), such that the system is restricted to the slow manifold: $M_{1} x+M_{2} z=0$. It follows that this algebraic equation has an analytical solution given by $H(x)=-M_{2}^{-1} M_{1} x$. Hence, Assumption 2 holds. Then, we obtain that the slow model is defined by

$$
\begin{aligned}
\dot{x} & =A x+\bar{f}(t, x), \\
y_{s} & =C x+D w,
\end{aligned}
$$

with $\bar{f}(t, x)=f\left(t, x,-M_{2}^{-1} M_{1} x\right), C=C_{1}-C_{2} M_{2}^{-1} M_{1} x$. Assumption 11. The function $\bar{f}(t, x)=f(t, x, H(x))$ in (33a) satisfies

$\bar{f}(t, x)=\left[\bar{f}_{1}\left(t, x_{1}\right), \bar{f}_{2}\left(t, x_{1}, x_{2}\right), \ldots, \bar{f}_{n}\left(t, x_{1}, \ldots, x_{n}\right)\right]^{T}$.

Moreover, $f(t, x, H(x))$ satisfies [Assumption 1, 6].

We need to state condition (34) to guarantee that it is possible to design an observer for the reduced system (33). Assumption 12. The reduced system (33) is globally exponentially practically stable.

It follows from Assumption 12 that Assumption 3 holds. Now, define the change of variables $z=\xi-M_{2}^{-1} M_{1} x$. Therefore, we have

$$
\begin{aligned}
\dot{x} & =A x+f\left(t, x, \xi-M_{2}^{-1} M_{1} x\right), \\
\epsilon \dot{\xi} & =M_{2} \xi+\epsilon\left(M_{2}^{-1} M_{1}\right)[A x \\
& \left.+f\left(t, x, \xi-M_{2}^{-1} M_{1} x\right)\right], \\
y & =C x+C_{2} \xi+D w
\end{aligned}
$$

By using the fast time scale $\tau=t / \epsilon$, it follows that the boundary layer system is given by $d \xi / d \tau=M_{2} \xi$. Since $M_{2}$ is Hurwitz, we have from [Theorem 4.6, 9] that for any given positive definite symmetric matrix $Q_{\xi}$ there exists a positive definite symmetric matrix $P_{\xi}$ that satisfies the Lyapunov equation: $P_{\xi} M_{2}+M_{2}^{T} P_{\xi}=-Q_{\xi}$. Then, consider $W(\xi)=\xi^{T} P_{\xi} \xi$ as a candidate Lyapunov function for boundary layer system. It follows that Assumption 4 holds with $a_{1}=\lambda_{\min }\left\{P_{\xi}\right\}, a_{2}=\lambda_{\max }\left\{P_{\xi}\right\}, a_{3}=-\lambda_{\min }\left\{Q_{\xi}\right\}$, $a_{4}=2 \lambda_{\max }\left\{P_{\xi}\right\}, a_{5}=0$ and $a_{6}=0$.

We now check conditions in Assumptions 5 and 6. Note that $L_{0}=0$ and $L_{3}=0$ because the right-hand side of the 
system does not depend on $\epsilon$. Since [Assumption 1, 6] holds, there is $L_{h g}>0$ satisfying that assumption such that (14) holds with $L_{1}=L_{h g_{1}}$. Note that the fact that $f(t, x, z)$ vanishes at $(x, z)=(0,0)$ implies that $|f(t, x, H(x))| \leq L_{h g_{2}}$, then $L_{2}=L_{h g_{2}}$. The isolated solution $H(x)$ is a function of $x$; hence, $L_{4}=0, L_{5}=\left|M_{2}^{-1} M_{1}\right|$ and $L_{6}=0$. Therefore, Assumptions 5 and 6 hold.

1) Observer design: Consider the high gain full-order observer presented in [6],

$$
\dot{\hat{x}}=A \hat{x}+\bar{f}(t, \hat{x})+G(\gamma, K)(y-C \hat{x}),
$$

where $\hat{x} \in \mathbb{R}^{n}$ is the observer's state and an estimate of the state, and $G(\gamma, K):=\left[\gamma_{1} k_{1}, \gamma_{2} k_{2}, \ldots, \gamma_{n} k_{n}\right]^{T}$ where $\gamma_{i}$ is the $i$-th component of $\gamma \in \mathbb{R}^{n}$ and $K=\left[k_{1}, k_{2}, \ldots, k_{n}\right]^{T}$ with $k_{i} \in \mathbb{R}, i=1, \ldots, n$ to be suitably chosen. Define the estimation error $\hat{e}=x-\hat{x}$, but as in [6], the stability of the estimation error is studied through the transformed coordinates $T(\gamma) e=\hat{e}$ where $T(\gamma)=\operatorname{diag}\left(\gamma_{1}, \gamma_{2}, \ldots, \gamma_{n}\right)$. It is well know that a linear transformation preserves the stability property of the system. The error dynamics in the transformed coordinates if defined as follows

$$
\begin{aligned}
\dot{e} & =\gamma_{1}(A-K C+\Omega(\gamma)) e(t)+T(\gamma)^{-1}(\bar{f}(t, x(t) \\
& -f(t, x(t)-T(\gamma) e(t))+T(\gamma)^{-1} G(\gamma, K) D w
\end{aligned}
$$

where $\Omega(\gamma)$ is a matrix given by

$$
\Omega:=\left[\begin{array}{ccccc}
0 & \alpha_{1} & 0 & \ldots & 0 \\
0 & 0 & \alpha_{2} & \ldots & 0 \\
\vdots & \vdots & \vdots & \vdots & \vdots \\
0 & 0 & 0 & \ldots & \alpha_{n-1} \\
0 & 0 & 0 & \ldots & 0
\end{array}\right],
$$

where $\alpha_{i}=\gamma_{i+1} /\left(\gamma_{1} \gamma_{i}\right)-1, i=1, \ldots, n-1$. From the definitions of $G(\gamma, K)$ and $T(\gamma)$, we can conclude that $T(\gamma)^{-1} G(\gamma, K) D w=K D w$. Therefore, (37) becomes

$$
\begin{aligned}
\dot{e} & =\gamma_{1}(A-K C+\Omega(\gamma)) e(t)+T(\gamma)^{-1}(\bar{f}(t, x(t) \\
& -f(t, x(t)-T(\gamma) e(t))+K D w
\end{aligned}
$$

We now consider the observer design in [6] in the absence of measurement noise. From [Theorem 2, 6], we know that if there exists a constant $\lambda>0$ and matrices $P=P^{T}>0$, $Y, S=S^{T}>0$ diagonal, and $W \leq 0$ diagonal such that

$$
\begin{aligned}
& {\left[\begin{array}{cc}
A^{T} P+P A+-C^{T} Y^{T}-Y C+\lambda I & \star \\
A_{1}^{T} P+W A_{2} & -2 S
\end{array}\right]<0,} \\
& W>-S
\end{aligned}
$$

and $\gamma$ is chosen such that

$$
\gamma_{1} \geq \frac{1}{\lambda_{\min }\left(S^{-1} W\right)+1}, \quad \gamma_{1}>\frac{2 k_{f} \lambda_{\max }(P)}{\lambda},
$$

with $k_{f}=\sqrt{\frac{n(n+1)}{2}} \max _{i=1,2, \ldots, n} L_{i}$ where $L_{i}$ are Lipschitz constants that come from [Assumption 1, 6], we can chose the gains as $K=P^{-1} Y$ and $\gamma_{i}=\gamma_{1}^{i} \prod_{k=1}^{i-1}\left(\alpha_{k}+1\right)$, $(i=2, \ldots, n)$. Since the observer must be implemented in the system with measurement noise, consider $V(e)=e^{T} P e$ as a Lyapunov function for (39). Then, if the stated above holds, we have

$$
\frac{\partial V_{2}}{\partial e} f_{e}(e) \leq-\nu|e|^{2}+2|e||w||K D| \lambda_{\max }\{P\},
$$

where $\nu>\gamma_{1}-2 k_{f} \lambda_{\max }\{P\} / \lambda>0$ and $f_{e}(e)$ is given by the right-hand side of (39). By applying completion of squares to (42), it follows that Assumption 7 holds with $a_{1}=$ $\lambda_{\min }\{P\}, a_{2}=\lambda_{\max }\{P\}, a_{3}=\nu, a_{4}=2 \frac{|K D|^{2} \lambda_{\max }\{P\}^{2}}{\nu}$ and $a_{4}=2 \lambda_{\max }\{P\}$. Note that the output of the system does not depend on $\epsilon$, then $L_{7}=0$ and it is straightforward to see that $L_{8}=|G(\gamma, K)|\left|C_{2}\right|$. Therefore, Assumption 8 also holds and our results apply and hold for this class of plants and observers. Notice that $L_{8}$ depends on $\gamma$, then a larger $\gamma$ would lead to more conservative results and a smaller $\epsilon^{*}$ would be required for our results to hold.

\section{B. Circle Criterion-based $\mathcal{H}_{\infty}$ observer}

We now consider the class of plants with a singularly perturbed structure such that the slow model has a form in which results from [7] can be applied,

$$
\begin{aligned}
\dot{x} & =A x+G \gamma(x)+B z, \\
\epsilon \dot{z} & =M_{1} x+M_{2} z, \\
y & =C_{1} x+C_{2} z+D w,
\end{aligned}
$$

where $x \in \mathbb{R}^{n}$ is the slow state, $z \in \mathbb{R}^{m}$ is the fast state, $y \in \mathbb{R}^{p}$ is the measured output variable, $w \in \mathbb{R}^{p}$ is the measurement noise which is $\mathcal{L}_{2}$ bounded, $\epsilon$ is the perturbation parameter of the process, $\gamma: \mathbb{R}^{n} \rightarrow \mathbb{R}^{q}$ is assumed to be globally Lipschitz and $A, B, G, C_{1}, C_{2}, D$, $M_{1}$ and $M_{2}$ are matrices of appropriate dimensions. The function $\gamma(\cdot)$ has the form

$$
\gamma(x)=\left[\gamma_{1}\left(F_{1} x\right), \ldots, \gamma_{i}\left(F_{i} x\right), \ldots, \gamma_{m}\left(F_{m} x\right)\right]^{T},
$$

where $F_{i} \in \mathbb{R}^{n_{i} \times n}$. The $n_{i}$ represents the number of rows of $F_{i}$ are not constrained [7]. By having a linear fast dynamics, we guarantee that the reduced model has the appropriate structure for which we can design the nonlinear observer [7]. Assumption 13. The matrix $M_{2}$ in (43b) is Hurwitz.

The above assumption is elemental since the approximation of the singularly perturbed system (43) through lower dimensional systems is only possible if $M_{2}$ is Hurwitz. Note that the system (43) does not have inputs, then Assumption 1 trivially holds. We now set $\epsilon=0$ in (43b), such that we obtain that $H(x)=-M_{2}^{-1} M_{1} x$. Hence, Assumption 2 holds. Note that the slow model is defined by

$$
\begin{aligned}
\dot{x} & =A_{0} x+G \gamma(x), \\
y_{s} & =C x+D w,
\end{aligned}
$$

where $A_{0}=A-B M_{2}^{-1} M_{1}$ and $C=C_{1}-C_{2} M_{2}^{-1} M_{1}$. Assumption 14. The reduced system (45) is Input-to-State practical stable.

By virtue of Assumption 14, there is a Lyapunov function such that the reduced system satisfies Assumption 3. This assumption gives generality to the plant since there is no need 
for $A_{0}$ to be Hurwitz. Now, define the change of variables $z=\xi-M_{2}^{-1} M_{1} x$. Therefore, we have

$$
\begin{aligned}
\dot{x} & =A_{0} x+G \gamma(x)+B \xi, \\
\epsilon \dot{\xi} & =M_{2} \xi+\epsilon\left(M_{2}^{-1} M_{1}\right)\left[A_{0} x+G \gamma(x)+B \xi\right], \\
y & =C x+C_{2} \xi+D w
\end{aligned}
$$

Following the exact same method as in the previous section, we have that the boundary layer system is defined by $d \xi / d \tau=M_{2} \xi$ where $\tau=t / \epsilon$. So, we can check that Assumption 4 holds with $a_{1}=\lambda_{\min }\left\{P_{\xi}\right\}, a_{2}=\lambda_{\max }\left\{P_{\xi}\right\}$, $a_{3}=-\lambda_{\min }\left\{Q_{\xi}\right\}, a_{4}=2 \lambda_{\max }\left\{P_{\xi}\right\}, a_{5}=0$ and $a_{6}=0$.

We now check the interconnection conditions in Assumptions 5 and 6 . The right-hand side of the system does not depend on $\epsilon$, then we have that $L_{0}=0$, and $L_{3}=0$. It follows from (45a) and (46a) that $L_{1}=|B|$. The function $\gamma(\cdot)$ is globally Lipschitz and it is observed that it vanishes at $x=0$, then there is a Lipschitz constant $L_{\gamma}>0$ such that $|G \gamma(x)| \leq L_{\gamma}|x|$. Moreover, we have that $\left|A_{0} x\right| \leq\left|A_{0}\right||x|$, so we have that $\left|A_{0} x+G \gamma(x)\right| \leq\left(L_{\gamma}+\left|A_{0}\right|\right)|x|$. Then, $L_{2}=L_{\gamma}+\left|A_{0}\right|$. Since the isolated solution $H(x)$ is a function of $x$ we have $L_{4}=0, L_{5}=\left|M_{2}^{-1} M_{1}\right|$ and $L_{6}=0$.

1) Observer design: Consider the generalized circle criterion observer proposed in [7] which has the following form

$$
\dot{\hat{x}}=A_{0} \hat{x}+G\left[\gamma_{1}\left(\hat{v}_{1}\right), \ldots, \gamma_{m}\left(\hat{v}_{m}\right)\right]^{T}+L\left(y_{s}-C \hat{x}\right)
$$

with $\hat{v}_{i}=H_{i} \hat{x}+K_{i}\left(y_{s}-C \hat{x}\right)$ where $i \in\{1, \ldots, m\}$, $\hat{x} \in \mathbb{R}^{n}$ is the observer's state and an estimate of the slow state, $K_{i}$ and $L$ are gain matrices of appropriate dimensions which must be designed. We design the observer (47) for the reduced system (45). Define the estimation error as $e:=x-\hat{x}$. By considering [Lemma 2, 7] and the features of the system such as the global Lipschitz property of $\gamma(\cdot)$ among other conditions, it is shown in [7] that the error dynamics is defined is given by

$$
\dot{e}=(A-L C+\mathbb{A}) e+(-L D+\mathbb{B}) w
$$

where $\mathbb{A}=\sum_{i, j=1}^{i, j=m, n_{i}}\left[\phi_{i j} G \mathcal{H}_{i j} \mathbb{F}_{K_{i}}\right]$ and $\mathbb{B}=$ $\sum_{i, j=1}^{i, j=m, n_{i}}\left[\phi_{i j} G \mathcal{H}_{i j} \mathbb{D}_{K_{i}}\right]$, in which $\mathcal{H}_{i j}=\hat{\iota}_{m}(i) \hat{\iota}_{n_{i}}^{T}(j)$, $\mathbb{H}_{K_{i}}=H_{i}-K_{i} C, \mathbb{D}_{K_{i}}=-K_{i} D$, where $\hat{\iota}_{s}(i)$ is a vector of the canonical basis of $\mathbb{R}^{s}$ with the element 1 in the $i$ th position, the functions $\phi_{i j}$ are defined by [Lemma 2, 7] (see [7] for further details). The observer design problem is to determine the parameters such that the $\mathcal{H}_{\infty}$ criterion $|e|_{\mathcal{L}_{2}}^{2} \leq \rho\left|e_{0}\right|^{2}+\mu|w|_{\mathcal{L}_{2}}^{2}$ is satisfied and $\mu$ is minimised. [Theorem 4, 7] gives a LMI that must be solved to minimize $\mu$ and to design the observer's gain matrices $L$ and $K_{i}$. Moreover, the solution of the LMI condition gives a symmetric positive definite matrix $P \in \mathbb{R}^{n \times n}$ such that $V_{2}(e)=e^{T} P e$ is a Lyapunov function for (48). If $P$ is a solution for the LMI in [Theorem 4, 7], its derivative satisfies

$$
\frac{\partial V_{2}}{\partial e} f_{e}(e, w) \leq-|e|^{2}+\mu|w|^{2},
$$

where $f_{e}(e, w)$ is given by the right-hand side of (48). It is observed that (49) implies the $\mathcal{H}_{\infty}$ criterion is satisfied with $\rho=\lambda_{\max }\{P\}$. Then, Assumption 7 holds with $a_{1}=\lambda_{\min }\{P\}, a_{2}=\lambda_{\max }\{P\}, a_{3}=1, a_{4}=\mu$ and $a_{5}=\lambda_{\max }\{P\}$. We now look at the observer dynamics to verify Assumption 8. Since the output of the system does not depend on $\epsilon$ we have $L_{7}=0$. On the other hand, (29) is satisfied with $L_{8}=\left|L C_{2}\right|+|G|\left|\sum_{i} K_{i} C_{2}\right|$. Therefore, Assumptions 1 - 8, Lemma 1 and Theorem 1 hold.

\section{CONClusions}

We proved the robustness properties of nonlinear observers with respect to measurement noise and fast dynamics in singularly perturbed models. As far as we are aware, this is the first time that measurement noise is considered in the singular perturbation framework for observer design. We showed that the error dynamics exhibit practical ISS and practical $\mathcal{L}_{2}$ stability properties. From our main result, we can conclude that the estimation error globally practically converges in the absence of measurement noise which is a stronger result than the one in [1]. Whilst semi-global results are presented in [1] and [2], we concluded global stability under stronger assumptions in this work. Although the observer design process does not consider the fast variables, we showed that the observer performs well when implemented on the full system. Our methodology is not constructive, but instead, it is prescriptive as it outlines the conditions on the system and observer that would guarantee good performance of observers for slow variables that were designed base on the reduced (slow) model.

\section{REFERENCES}

[1] L. Cuevas, D. Nešić, and C. Manzie, "Convergence of full-order observers for the slow states of a singularly perturbed system (Part I: Theory)," 2018 Australian and New Zealand Control Conference (ANZCC), Melbourne, VIC, 2018, to be published.

[2] L. Cuevas, D. Nešić, and C. Manzie, "Convergence of full-order observers for the slow states of a singularly perturbed system (Part II: Applications)," 2018 Australian and New Zealand Control Conference (ANZCC), Melbourne, VIC, 2018, to be published.

[3] N. Kazantzis, N. Huynh, and R. A. Wright, "Nonlinear observer design for the slow states of a singularly perturbed system," Computers and Chemical Engineering, vol. 29, no. 4, pp. 797-806, 2005.

[4] R. Castro-Linares, J. Alvarez-Gallegos, and V. Vazquez-Lopez, "Sliding Mode Control and State Estimation for Class of Nonlinear Singularly Perturbed Systems," Dynamics and Control, vol. 11, no. 1, pp. 25-46, 2001.

[5] Y. Wang, and W. Liu, "Robust Observer-Based Feedback Control for Lipschitz Singularly Perturbed Systems," Mathematical Problems in Engineering, 2015.

[6] A. Alessandri, and A. Zemouche, "On the Enhancement of High-Gain Observers for State Estimation of Nonlinear Systems," Proceedings of the 55th Conference on Decision and Control, pp. 6278-6283, 2016.

[7] A. Zemouche, R. Rajamani, G. Phanomchoeng, B. Boulkroune, H. Rafaralahy, and M. Zasadzinski, "Circle criterion-based $\mathcal{H}_{\infty}$ observer design for Lipschitz and monotonic nonlinear systems-Enhanced LMI conditions and constructive discussions," Automatica, vol. 85, pp. 412425, 2017.

[8] P. D. Christofides, and A. R. Teel, "Singular Perturbations and Inputto-State Stability," IEEE Transactions on Automatic Control, vol. 41, no. 11, pp. 1645-1650, 1996.

[9] H. K. Khalil, Nonlinear Systems (3rd ed.). New Jersey: Prentice Hall, 2001.

[10] D. Nešić, and P. Dower, "A Note on Input-to-State Stability and Averaging of Systems with Inputs," IEEE Transactions on Automatic Control, vol. 46, no. 11, pp. 1760-1765, 2001.

[11] M. Corless, and L. Glielmo, "On the Exponential Stability of Singularly Perturbed Systems," In SIAM Journal on Control and Optimization, vol. 30, no. 6, pp. 1338-1360, 1991. 


\section{University Library}

\section{- M I I N E R VA \\ A gateway to Melbourne's research publications}

Minerva Access is the Institutional Repository of The University of Melbourne

Author/s:

Cuevas, L;Nesic, D;Manzie, C

Title:

Global stability of the error dynamics of an observer designed for the slow states of a singularly perturbed system

Date:

2018

Citation:

Cuevas, L., Nesic, D. \& Manzie, C. (2018). Global stability of the error dynamics of an observer designed for the slow states of a singularly perturbed system. Proceedings of the 2018 15th International Conference on Control, Automation, Robotics and Vision (ICARCV), 00, pp.698-703. IEEE. https://doi.org/10.1109/ICARCV.2018.8581224.

Persistent Link:

http://hdl.handle.net/11343/251883 